11 | 2007

Varia

\title{
L'inventaire des châteaux bourguignons : bilan et perspectives
}

Hervé Mouillebouche

\section{(2) OpenEdition}

1 Journals

Édition électronique

URL : https://journals.openedition.org/cem/1097

DOI : 10.4000/cem.1097

ISSN : 1954-3093

Éditeur

Centre d'études médiévales Saint-Germain d'Auxerre

Édition imprimée

Date de publication : 15 août 2007

ISSN : 1623-5770

Référence électronique

Hervé Mouillebouche, "L'inventaire des châteaux bourguignons : bilan et perspectives », Bulletin du centre d'études médiévales d'Auxerre | BUCEMA [En ligne], 11 | 2007, mis en ligne le 30 août 2007,

consulté le 22 septembre 2022. URL : http://journals.openedition.org/cem/1097 ; DOI : https://doi.org/ 10.4000/cem. 1097

Ce document a été généré automatiquement le 22 septembre 2022.

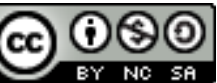

Creative Commons - Attribution - Pas d'Utilisation Commerciale - Partage dans les Mêmes Conditions 4.0 International - CC BY-NC-SA 4.0

https://creativecommons.org/licenses/by-nc-sa/4.0/ 


\title{
L'inventaire des châteaux bourguignons : bilan et perspectives
}

\author{
Hervé Mouillebouche
}

1 La castellologie bourguignonne se porte bien. Depuis de nombreuses années, les opérations de fouilles, de prospections ou d'études de bâti se sont multipliées sur les quatre départements de Bourgogne, grâce notamment au dynamisme des associations de bénévoles ${ }^{1}$. Cette effervescence a naturellement conduit à l'arrivée de plusieurs castellologues dans les rangs d'ARTeHIS UMR 5594. Cinq chercheurs: Hervé Mouillebouche, Vasco Zara, Michel Maerten, Emmanuel Laborier et Fabrice Cayot ${ }^{2}$ unissent aujourd'hui leurs efforts autour de quelques lignes directrices pour mener plus loin l'étude des châteaux bourguignons.

2 Les vestiges d'habitats fortifiés sont une source essentielle pour étudier le Moyen Âge. En effet, l'habitat est une image de l'habitant. Le château, la maison forte sont beaucoup plus que des lieux de défense. Ce sont aussi des moyens d'expression du pouvoir ; plutôt qu'à contrôler le territoire, ils servent surtout à imposer une marque visuelle sur le paysage. Le château est un instrument de communication, il délivre plus ou moins consciemment - un message.

3 Dès lors, il appartient aux médiévistes d'une part d'étudier ces vestiges pour apprendre à décrypter ces messages, d'autre part - et avant tout - de les répertorier, de les décrire et de les éditer, afin que cette documentation soit accessible aux chercheurs étrangers, mais aussi aux chercheurs futurs. Les axes d'études que nous avons retenus pour les années à venir vont dans ce double sens d'inventorier et de comprendre.

Un logiciel développé pour l'étude du patrimoine monumental médiéval 


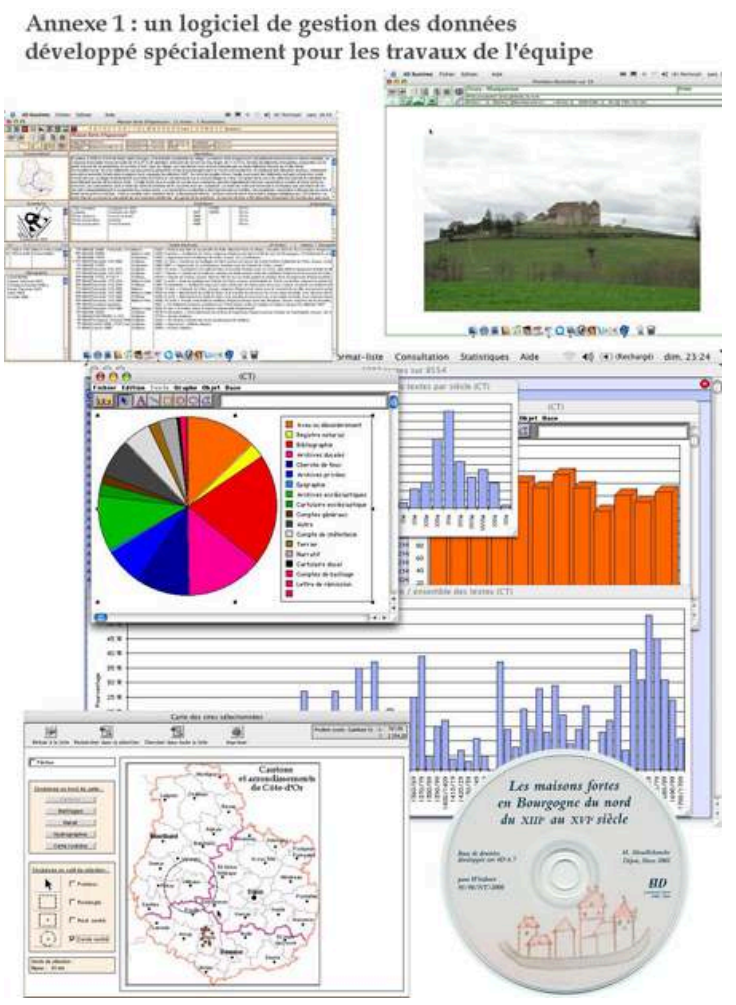

4 À l'occasion de précédentes études sur les châteaux, nous avons développé un logiciel de gestion de données très sophistiqué (sous $4 \mathrm{D}$ ), qui permet d'optimiser le travail de collectes de données et de recherches. Ce logiciel est destiné tout autant au grand public qu'aux chercheurs, puisqu'il permet aussi bien une consultation facile de la documentation qu'une utilisation pointue des outils de recherche et de calcul. Il évolue continuellement, depuis sa création en 1992, en fonction de nouvelles questions ou des nouvelles utilisations qui en sont faites.

La base de données, qui gère simultanément des textes, des images et des données alphanumériques, est constituée de 6 fichiers périphériques : Communes, Textes, Images, Prosopographie, Bibliographie, États séquentiels, qui sont reliés au fichier principal contenant les fiches de sites. Tous les fichiers sont interdépendants : ils peuvent être consultés un par un, et toutes les fiches liées à un même site sont consultables à partir de la fiche du site. Les requêtes peuvent se faire aussi en croisant les fichiers sur plusieurs niveaux: on peut par exemple chercher les communes qui contiennent un site qui, d'une part, a connu un état séquentiel d'occupation en 1474, et d'autre part est documenté par un texte de la série B dont un des protagonistes est une femme noble. On peut aussi faire des requêtes plus simples.

6 Le logiciel a connu une première diffusion en 2002 avec l'ouvrage d'Hervé Mouillebouche sur les maisons fortes ${ }^{3}$. Depuis, il a considérablement évolué, par l'adjonction de fonctions intégrées d'aide à la recherche et à la saisie.

- Générateur automatique de graphes. Le générateur de graphes natif du programme 4D permet de réaliser rapidement et simplement la plupart des requêtes courantes. Néanmoins, il nous a paru peu à peu nécessaire de pouvoir disposer instantanément de certains graphes très utiles, notamment celui des évolutions chronologiques. Ce graphe est désormais disponible en menu dans tous les fichiers. Une programmation plus poussée permet également d'obtenir des « pyramides démographiques » des forteresses : le programme dénombre, pour 
chaque intervalle de temps défini, le nombre de sites en activité (c'est-à-dire le nombre de sites apparus avant la fin de la période, moins le nombre de sites détruits avant le début de la période), pour en faire des diagrammes en barre, imprimables ou exportables.

- Gestionnaire d'images. L'étude des châteaux nécessite la manipulation d'un grand nombre d'images : photos de prospection, photos aériennes, relevés, plans anciens, plans d'archives, etc., toutes ces photos peuvent être stockées dans la base de données. Primitivement, elles pouvaient être recherchées par site, par région, ou par un certain nombre de critères enregistrés. Le dernier développement de la base de données propose d'une part une fonction " planche contact », qui affiche sur le même écran les vignettes des images d'une sélection donnée, d'autre part une fonction de diaporama, qui permet de faire défiler, manuellement ou automatiquement, une sélection d'images. C'est en quelque sorte IPhoto et Powerpoint réinventés sous 4D. Un module de comparaison permet de garder plusieurs images simultanément à l'écran, éventuellement en les réduisant. Enfin, un module de mesure à l'écran permet d'obtenir la distance et l'angle entre deux points : distance à l'écran, sur papier, ou distance réelle si la mesure s'effectue sur un plan à l'échelle.

- Système d'information géographique. Les sites, les communes, mais aussi les états séquentiels, les images et les textes sont géo-référencés. Ils peuvent être présentés sur la carte de leur département, avec différents fonds de carte. Un passage de la souris sur un point fait apparaître le nom du site. Mais le module de cartographie permet également des recherches par zone, pour trouver par exemple tous les textes compris dans un rectangle de $100 \mathrm{~km}$ de côté, ou toutes les photographies aériennes dans un rayon de $15 \mathrm{~km}$ autour d'Auxerre. La base de données intègre aussi les coordonnées du centre de chaque commune, ce qui permet de présenter à l'écran la répartition des sites (et des faux sites) d'une commune, et de calculer automatiquement la distance et la direction d'un site par rapport au centre de sa commune.

- Système d'indexation automatique. Pour être manipulables facilement, les textes doivent être indexés avec des clefs d'entrées préenregistrées. Ainsi, pour travailler sur les arrières fiefs, au lieu de rechercher « Texte / Contient / « arrière-fief » ou « arrière fief » ou « arrières fief@ » ou « rere fief » ou « rière fief » ou « rere fied » ou « retro feudum » ou « retro foedum » ou... bref, vous avez compris ", il suffit de chercher " Mot indexé / Est égal à / " arrièrefief ». Jusqu'à présent, l'indexation des textes se faisait manuellement, ce qui était long et pouvait conduire à des oublis. Désormais, une fonction préenregistrée analyse le vocabulaire du texte, en français, ancien français et latin, pour créer la liste des mots indexés.

Prochainement, cette fonction devrait s'étendre à une indexation automatique des personnes citées dans le texte, par une recherche des occurrences des prénoms les plus courants, avec une reconnaissance des noms et des titres.

- Communication et connectivité. Le logiciel 4D est réputé pour sa très grande capacité de communication avec les autres bases de données et les fonctions Internet. Actuellement, nous n'avons pas encore développé de fonctions de communications intégrées dans notre logiciel. L'échange de fichiers peut néanmoins se faire par la fonction d'importation/ exportation sous différents formats de tableurs. Ainsi, nous avons pu facilement importer des données en provenance de classeurs Excel, et exporter des sites datés et géo-référencés vers Arcview (CESRI), ArcGis (CESRI) ou Carto Explorer (@Bayo). Plus simplement, le logiciel offre de nombreux formats d'impression et de création de PDF.

7 Ce logiciel, qui a été développé pour l'étude des forteresses de la Côte-d'Or, est aujourd'hui opérationnel pour les quatre départements de la Bourgogne, et pourra être étendu à d'autres départements si l'occasion se présente. Le logiciel a également été adapté pour être utilisé pour d'autres inventaires de vestiges médiévaux. En changeant 
quelques noms de champs, il a pu être utilisé pour l'inventaire des hôpitaux médiévaux et pour celui des plates-tombes médiévales ${ }^{4}$.

Un inventaire en cours d'élaboration

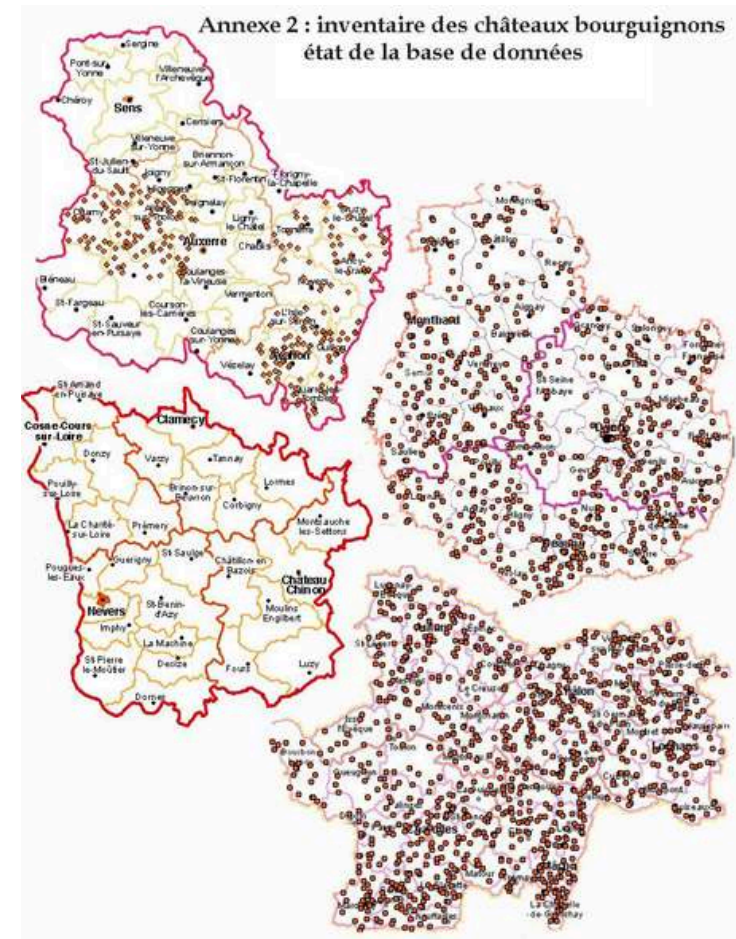

8 Il n'existe, en Bourgogne comme ailleurs, aucun inventaire exhaustif des châteaux. Les publications qui s'accumulent depuis plusieurs siècles ne prennent jamais en compte l'ensemble des vestiges (de pierre ou de terre). Bien pire : cette longue historiographie a fait naître nombre de châteaux imaginaires, qui n'ont aucune existence historique. Un inventaire scientifique doit donc s'accompagner d'une vérification systématique sur le terrain, avec un relevé et une description précise des vestiges. Cet inventaire est inégalement mené sur les quatre départements de la Bourgogne.

- En Côte-d'Or, l'inventaire a été mené par H. Mouillebouche de 1986 à 2002 ; il a été publié, sous forme d'un livre et d'un cédérom, aux Éditions Universitaires de Dijon en 2002. (664 sites) ${ }^{5}$. Il se perfectionne lentement, au gré des découvertes et des nouvelles collaborations. Il comprend actuellement 8500 textes ou mentions d'archives, 3220 images dont 610 plans normalisés au 1/1000.

- En Saône-et-Loire, l'inventaire a été mené de 1994 à 2004 par de Centre de Castellologie de Bourgogne ( $\mathrm{CeCaB}$ ) sous la direction de Michel Maerten. Il a été déposé au Service régional de l'archéologie sous la forme de 27 volumes papier. Ce travail est en cours de saisie sur la base de données. Il compte actuellement 1026 sites, 290 faux sites, 3500 textes et 3200 illustrations dont 591 plans normalisés.

- Dans la Nièvre, l'inventaire a été réalisé en grande partie par Brigitte Colas en $1994^{6}$. L'informatisation des données n'est plus qu'une question de temps (voire de moyens). - Dans l'Yonne, deux étudiants de maîtrise ont réalisé un inventaire de l'est de l'arrondissement d'Auxerre et du nord de l'arrondissement d'Avallon. Le sud de cet arrondissement est en cours de traitement par un étudiant de Master ${ }^{7}$. 
- Cette précieuse base de données n'est pas encore disponible sur Internet. Actuellement, elle fait l'objet d'une diffusion restreinte à tous les membres du Centre de Castellologie de

Bourgogne ${ }^{8}$.

Projets et axes d'études

9 Parallèlement à l'élaboration et la numérisation de la base de données, et en plus de leurs recherches personnelles, les castellologues d'ARTeHIS-UMR 5594 ont défini plusieurs axes de recherches collectives, qui tous mettent en œuvre l'exploitation de la base de données.

Standardisation des forteresses bourguignonnes

10 Les castellologues connaissent depuis longtemps le phénomène de standardisation des châteaux royaux. À la fin du XII ${ }^{\mathrm{e}}$ siècle Philippe Auguste, pour réaliser des économies de chantier, mais aussi pour imprimer une marque visible et reconnaissable de la présence royale, a demandé à ses architectes de construire un peu partout des tours «du module de la tour du Louvres » ${ }^{9}$. Ces châteaux philippiens se reconnaissent aussi par leur rigueur symétrique: plan carré cantonné de tours rondes, tour maîtresse indépendante, porte flanquée de tours rondes, sans pont-levis. Au milieu du XIII ${ }^{\mathrm{e}}$ siècle, un autre standard de fortification apparaît en Suisse Romande : le "carré savoyard " ${ }^{10}$. Il s'agit d'un plan carré cantonné plus petit, plus trapu, entouré de fausses braies (type Yverdon) ${ }^{11}$. Or, pour certains historiens, les origines de ces deux modèles pourraient être bourguignonnes: Druyes-les-Belles-Fontaines pourrait être le prototype des châteaux philippiens; Semur-en-Auxois celui des carrés savoyards ${ }^{12}$. Mais ces hypothèses restent fragiles, parce que les châteaux bourguignons sont mal datés. Ce serait donc un signalé service à rendre à la communauté scientifique que de pouvoir dater plus précisément ces structures, notamment par la dendrochronologie.

11 La standardisation est également illustrée par l'abondance des tours carrées à baiescréneaux. 


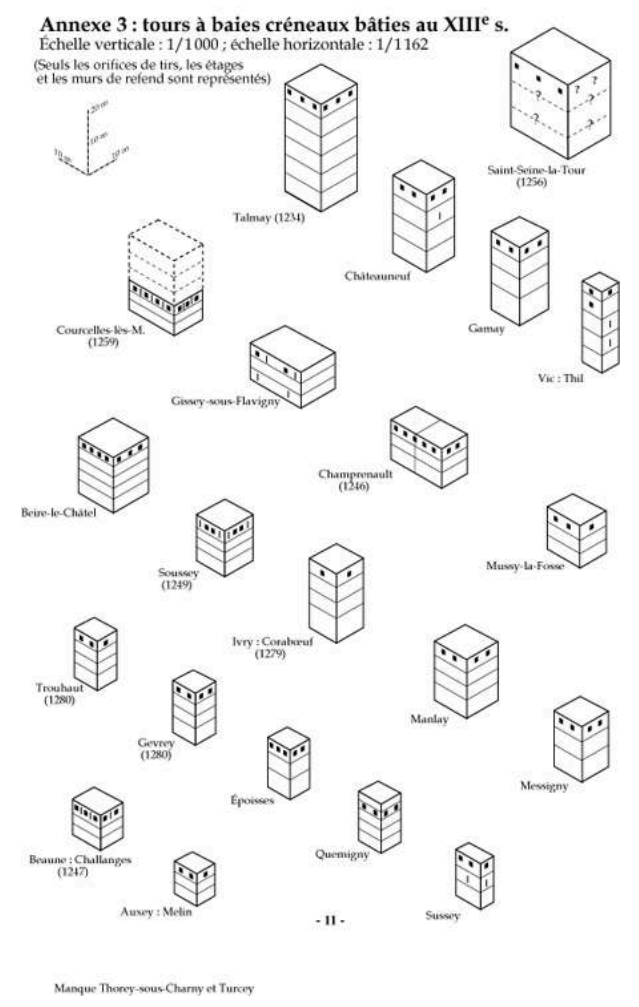

Ces tours, nombreuses en Bourgogne, sont souvent datées par une première mention remontant à la première moitié du XIII ${ }^{e}$ siècle. Certains castellologues, notamment de l'école alsacienne, pensent que les vestiges sont antérieurs aux textes et qu'il faut les dater du XII ${ }^{e}$ siècle ${ }^{13}$. En revanche, les chercheurs de l'école suisse affirment que les baies-créneaux ne peuvent pas être antérieures à 1250 ; les mentions de forteresses avant cette date correspondraient donc à un premier état disparu ${ }^{14}$. Enfin, tout le monde s'accorde à reconnaître que Cluny, à travers ses fortifications, mais aussi à travers ses prieurés, a eu un grand rôle dans la diffusion de ces modèles. La belle étude de Fr. Didier sur la tour ronde de Cluny constitue une étape intéressante qu'il est important de renouveler ${ }^{15}$.

Aristocratie et motte castrales, du Xe au XII ${ }^{\mathrm{e}}$ siècle.

Un historien a récemment évoqué, pour qualifier la castralisation du $\mathrm{XI}^{\mathrm{e}}$ siècle, l'image d'un «brun manteau de mottes " ${ }^{16}$. L'expression est belle, mais partiellement inexacte. Tout d'abord, parce que les mottes sont vertes. Surtout, parce qu'elles sont beaucoup moins nombreuses sur le terrain que dans la tapisserie de Bayeux et dans la bibliographie qui s'en inspire. Les inventaires menés en Bretagne, en Normandie ou en Bourbonnais, et qui ont pu accréditer l'idée d'un vaste mouvement d'emmottement, sont souvent des inventaires de chimères. Les inventaires plus rigoureux de Champagne, de Lorraine, de Savoie ${ }^{17}$ - et bien sûr de Bourgogne, montrent que les vraies mottes sont rares, et l'ont toujours été.

Pour comprendre le rôle spécifique de la motte castrale par rapport aux autres formes de fortifications précoces (enceinte, castrum, oppidum), il est nécessaire de recourir à l'archéologie et à l'étude des sources écrites.

En ces temps de vaches maigres pour les fouilles de sauvetage, et de vaches mortes pour les fouilles programmées, les archéologues bourguignons peuvent utiliser leurs 
loisirs forcés à relire et à publier les rapports des fouilles anciennes. Ainsi, deux castellologues de l'UMR ARTeHIS, Michel Maerten et Hervé Mouillebouche, ont été sollicités par le Groupe Archéologique du Mâconnais pour coordonner l'étude et l'édition des fouilles de la motte de Loisy. Ce travail a déjà montré le côté aristocratique, mais aussi atypique de l'alimentation des occupants ${ }^{18}$, alors qu'une analyse plus serrée des textes a permis de retrouver l'identité du bâtisseur de cette petite forteresse.

L'inventaire des sites de terre doit nécessairement s'accompagner de relevés topographiques. Après les relevés en coupe au niveau optique, les relevés en courbes de niveaux au théodolite, arrivent maintenant les relevés de troisième génération, au théodolite laser ou au DGPS, avec rendu en 3D et reconstitution virtuelle. De tels relevés ont déjà été effectués en Côte-d'Or, en collaboration avec le centre de Cartographie et de Géomatique de l'Université de Bourgogne, et en Saône-et-Loire par le Groupe de Recherche Archéologique de Tournus.

Enfin, cet inventaire de sites doit nécessairement être croisé avec les sources écrites, pour essayer de faire des recoupements entre les sites fortifiés et les anciennes familles aristocratiques. Ce travail, qui a été ébauché il y a cinquante ans par Georges Duby, avec des méthodes et des problématiques du siècle dernier, peut désormais être traité exhaustivement grâce d'une part à la base de données de l'habitat fortifié, d'autre part à la base de données des Chartes de la Bourgogne du Moyen Âge (CBMA, s. dir. E. Magnani et M.-J. Gasse-Grandjean).

Châteaux, mesures et harmonie

Quelles relations y a-t-il entre les châteaux, les nombres et la musique ? C'est tout un, répondra le médiéviste, qui sait bien qu'au Moyen Âge, l'harmonie et la musique soustendent tout discours spéculatif. Ces thématiques sont au cœur des préoccupations de Vasco Zara. Ce jeune musicologue italien, qui vient d'intégrer notre UMR, a mené une étude pionnière sur l'importance des proportions musicales dans l'architecture moderne et médiévale, et notamment dans les châteaux de Frédéric II ${ }^{19}$. Pour une telle étude, notre équipe pourra également bénéficier de la double compétence, en histoire et en musique, de Martine Clouzot, bien connue pour ses travaux sur les musiciens à la cour de Bourgogne ${ }^{20}$.

Une telle étude ne nécessitera pas d'énormes investissements, mais surtout un échange de méthodes et de compétences. En effet, la plupart des plans des châteaux sont déjà disponibles dans la base de données. Il s'agit maintenant de sélectionner les châteaux princiers ou ceux des grands féodaux qui semblent avoir bénéficié d'un plan savant ; on pourra reprendre s'il le faut quelques mesures sur le terrain ; puis il faudra voir si les plans-masses et les élévations ont pu - et dans quelle mesure - être dessinés et élevés selon des proportions calquées sur les harmonies musicales.

Les châteaux des Rolin

20 Les récents colloques et travaux collectifs sur Nicolas Rolin ont montré toute l'importance du chancelier dans la vie politique et artistique de la fin $\mathrm{du} \mathrm{XV} \mathrm{XV}^{\mathrm{e}}$ siele ${ }^{21}$ Curieusement, les châteaux de Nicolas Rolin et de ses héritiers n'ont guère retenu l'attention des chercheurs depuis le petit article, pourtant fort suggestif, de Fr. Vignier ${ }^{22}$. L'inventaire des biens de Nicolas Rolin, dressé à sa mort, fait état d'environ 50 bâtiments fortifiés, dont 30 sont situés en Bourgogne ${ }^{23}$. 


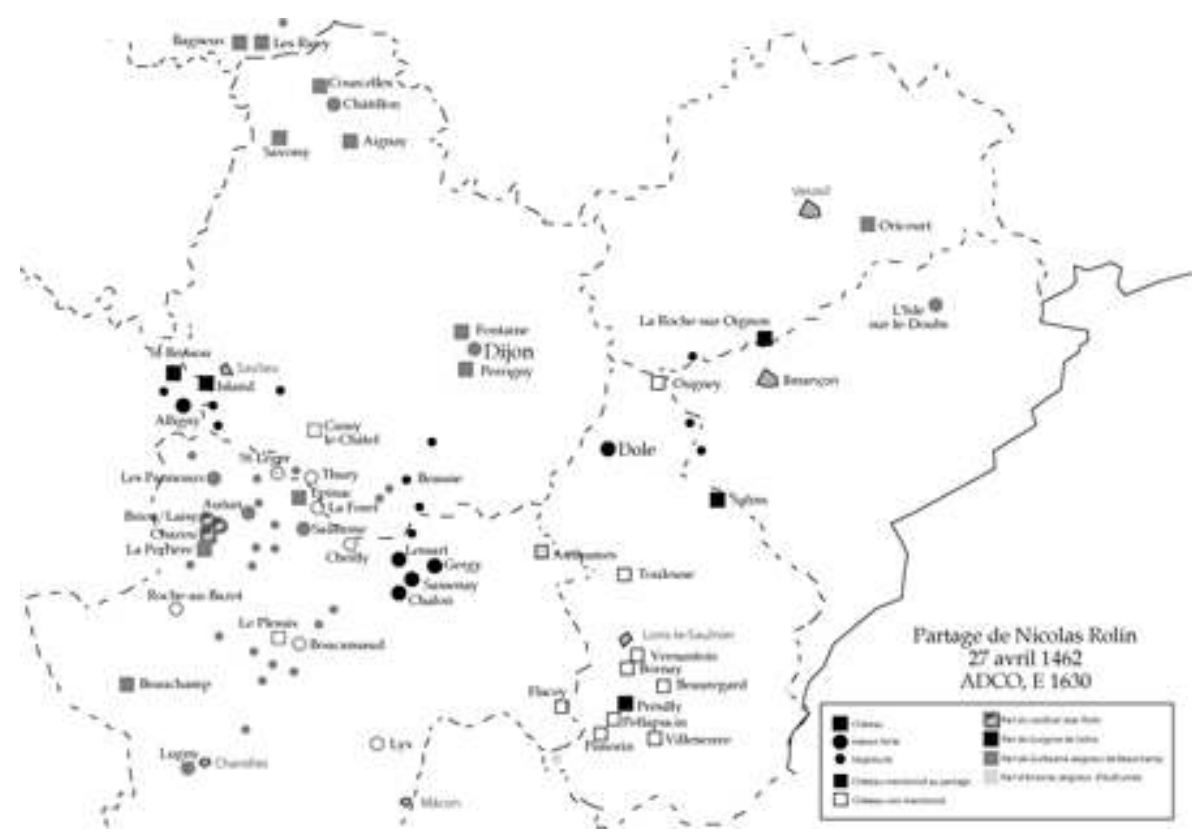

Parmi ces châteaux, certains, élevés du temps du chancelier lui-même, offrent encore des vestiges spectaculaires : c'est notamment le cas de Chazeu, Savoisy, ou Authumes ${ }^{24}$. Les châteaux de Présilly et de Perrigny, qui ont bénéficié de fouilles archéologiques, ont fourni du mobilier datant de l'époque du chancelier ${ }^{25}$. Une prochaine opération de "dégagement des murs " du château de La Perrière devrait également apporter des documents neufs sur le patrimoine des Rolin ${ }^{26}$.

Le Centre de Castellologie de Bourgogne et les castellologues de l'UMR prévoient d'éditer une étude globale de ces châteaux en 2010 environ. Cette étude comportera une monographie de chaque bâtiment et une étude plus fine, avec relevés, des principaux châteaux élevés par le chancelier. Cette publication se fera bien sûr en collaboration avec d'autres équipes de recherches, notamment pour étudier les châteaux de Franche-Comté et du Hainaut.

À priori, les châteaux de Nicolas Rolin posent deux séries de questions :

- La répartition : quelle était la politique d'acquisition du chancelier ? On voit bien sûr un intérêt particulier pour la région d'Autun. Mais s'agissait-il uniquement d'une opération de prestige, pour se donner des apparences de prince d'Autun, ou plutôt d'une opération stratégique, visant à contrôler la vallée de l'Arroux, et les accès de la Bourgogne du sud ? De même, les châteaux de Franche-Comté semblent plus répondre à des besoins de contrôle des voies qu'à une politique d'acquisition strictement immobilière.

- La construction : le chancelier a fait élever lui-même plusieurs résidences ; il fut dans ce domaine aussi dynamique que les derniers Valois. Les châteaux de Nicolas Rolin constituent l'une des plus grosses « collections » de forteresses $\mathrm{du} \mathrm{XV}$ e siècle, ce qui montre que le Chancelier s'était bel et bien lancé dans la grande compétition princière du règne de Charles VI. L'étude de ces bâtiments permettra donc de mieux connaître l'évolution de la castellologie en Bourgogne à la fin de la guerre de Cent Ans. En outre, on pourra essayer de voir si le chancelier a utilisé un ou plusieurs architectes, et si les mêmes équipes de maçons ont pu travailler sur plusieurs châteaux, et éventuellement sur ses hôtels de Dijon, Dôle et Autun, et sur l'Hôtel Dieu de Beaune. 
Bien sûr, cette dernière étude permettra d'intégrer les outils élaborés dans les trois études précédentes. Y a-t-il une recherche de standardisation dans les châteaux Rolin, et cette standardisation vient-elle des bâtisseurs, de la région, ou du commanditaire? Parmi ces possessions, le chancelier tenait en fief quelques anciennes mottes castrales (mottes d'Authumes, de Flacey). Ces mottes avaient-elles encore un sens, et un intérêt, au moins symbolique, au XV $\mathrm{XV}^{\mathrm{e}}$ siècle ? Enfin, ce grand mécène, ami des arts, est le type même du bâtisseur qui aurait pu chercher à respecter les règles universelles de l'harmonie dans la construction de ses hôtels et de ses châteaux. Nicolas Rolin est donc vraiment la pierre de touche qui permettra de vérifier la pertinence et la cohérence des recherches de notre équipe.

\section{NOTES DE FIN}

1. Les chercheurs sont notamment issus de ARHM Fort (Association de Recherche d'Histoire Médiévale sur les Fortifications ; GAM (Groupe Archéologique du Mesmontois) ; LPO (Le Patrimoine Oublié) ; Groupe PPGR (Groupe Pluridisciplinaires des Périodes Gothiques et Romanes). Une collaboration scientifique se poursuit avec le $\mathrm{CeCaB}$ (Centre de Castellologie de Bourgogne), le Groupe PPGR et LPO.

2. Hervé Mouillebouche, maître de conférences en histoire médiévale ; Vasco Zara, Maître de conférences en musicologie médiévale ; Michel Maerten, enseignant, docteur en archéologie médiévale ; Emmanuel Laborier, archéologue à l'INRAP, doctorant ; Fabrice Cayot, historien libéral, doctorant.

3. H. MOUILLEBOUCHE, Les maisons fortes en Bourgogne du nord, du XIII ${ }^{e}$ au XVI ${ }^{e}$ siècle, Dijon, 2002 ( 1 volume et 2 cédéroms).

4. Inventaire des édifices de charité médiévaux : thèse en cours par Aurore-Diane Simon, sous la direction d'A. Saint-Denis. (Voir A. SAINT-DENIS, « L'assistance en Bourgogne, $\mathrm{V}^{\mathrm{e}}-\mathrm{XV}^{\mathrm{e}} \mathrm{s}$. ", in Bulletin du Centre d'études médiévales, 9 (2005), p. 87-90. Inventaire des plates-tombes de Bourgogne : master en cours par Guillaume Grillon, sous la direction d'A Saint-Denis et H. Mouillebouche.

5. H. MOUILLEBOUCHE, op. cit.

6. B. COLAS, Vestige d'habitat seigneurial fortifié dans l'ouest Nivernais, $X I^{e}-X V I^{e}$ siècles. Dijon, 1994 (thèse de doctorat sous la direction de M. Bur, Nancy).

7. F. САYOT, Fortifications et habitats fortifiés dans l'ouest de l'Yonne, du XIe au XVI siècle, Mémoire de maîtrise d'histoire médiévale, sous la direction de V. Tabbagh, Université de Dijon, 1999. Th. LORAIN, L'habitat fortifié dans le Tonnerrois du XIII au XVI $s$, Mémoire de maîtrise d'histoire médiévale sous la direction d' A. Saint-Denis et H. Mouillebouche, Dijon, 2003. J. MARQUIS, L'habitat fortifié dans l'Avallonnais entre le XII et le XVI siècle (en cours), sous la direction d'H. Mouillebouche.

8. On peut adhérer au $\mathrm{CeCaB}$, et recevoir le cédérom de la Bourgogne, en contactant Gilles Auloy (105 av. de la Libération - 71210 Montchanin).

9. A. CHÂtELAIN, « Recherche sur les châteaux de Philippe Auguste », in Archéologie Médiévale, 21 (1991), p. 115-161. A. ERLANDE-BRANDENBURG, « L'architecture militaire au 
temps de Philippe Auguste : une nouvelle conception de la défense ", in La France de Philippe Auguste. Le temps des mutations, Paris, 1982, p. 595-603.

10. M. GRANDJEAN, Les monuments d'art et d'histoire du canton de Vaud. t. 4, Vaud, 1965.

11. D. de RAEMY, Châteaux, donjons et grandes tours dans les États de Savoie (1230-1330). Un modèle : le château d'Yverdon, Association pour la restauration du château d'Yverdon-lesBains, 2004 (Cahiers d'archéologie romande, 98).

12. Ch.-L. SALCH, Druyes-les-Belles-Fontaines; les châteaux sur plan carré dans l'Yonn, Strasbourg, 2001 (Châteaux forts d'Europe, $n^{\circ} 17$ ). D. de RAEMY, op. cit.

13. G. AULOY, Ch.-L. SALCH, Grands donjons romans en Bourgogne méridionale (Saône-et-Loire) $\mathrm{XI}^{e}$ et XII ${ }^{e}$ s., Strasbourg, 2005 (Châteaux forts d'Europe, n 32 (2004)).

14. D. de RAEMY, « L'architecture militaire : donjons et châteaux », in A. PARAVICINI (éd.), Les pays romands au Moyen Âge, Lausanne, 1997, p. 487-509.

15. Fr. DIDIER, « Cluny, la "tour ronde" de l'enceinte abbatiale », in Bulletin monumental, IV (2004), p. 312-316.

16. J. MORSEL, L'aristocratie médiévale, $V^{e}-X V^{e}$ siècle, Paris, 2004, p. 90.

17. M. BUR (dir.), Inventaire des sites archéologiques non monumentaux de Champagne, Reims, ARERS, 4 vol., 1972 à 1997. G. GIULIATTO, Châteaux et maisons fortes en Lorraine centrale. Paris, 1992 (Documents d'Archéologie Française, n 33). M. BOIS, M.-P. FEUILLET, P.Y. LAFFONT, CH. MAZART, J.-M. POISSON, E. SIROT, « Approche des plus anciennes formes castrales dans le royaume de Bourgogne-Provence ( $\mathrm{X}^{\mathrm{e}}-\mathrm{XII}^{\mathrm{e}} \mathrm{s}$.) », in Château-Gaillard, XVI (1994), p. 57-68.

18. J.-H. YVINEC, Étude archéo-zoologique du site de Loisy, à paraître.

19. V. ZARA, Musica e Architettura tra Medio Evo e Età moderna. Storia critica di un'idea, in Acta musicologica, . 77 (2005), p. 1-26. V. ZARA, Antichi e moderni tra musica e architettura : All'origine della « querelle des anciens et des modernes ", in Intersezioni, 26/2 (2006), p. 191-210. V. ZARA, L'intelletto armonico. Il linguaggio simbolico e musicale nell'architettura di Castel del Monte, in Musica et storia, 1 (2000), p. 15-52.

20. M. ClouzoT, Images de musiciens. 1350-1500. Figurations, typologies et pratiques sociales, Turnhout, 2007.

21. B. MAURICE-CHABARD ( dir.), La splendeur des Rolin : un mécénat privé à la Cour de Bourgogne, table ronde des 27-28 février 1995, Paris- Autun, 1999.

22. Fr. VIGNIER, «Les châteaux des Rolin », in Le faste des Rolin, au temps des ducs de Bourgogn,. Dijon, 1998 (Dossier de l'art, 49), p. 98-99.

23. ADCO, E 1630 ; voir aussi J.-B. DE VAIVRE, « La famille des Rolin », in La splendeur des Rolin, op. cit., p. 19-35. J. LAURENT, Les fiefs des Rolin. Compte rendu dans les Mémoires de la Société d'archéologie de Beaune, 1931-1932, p. 85-87.

24. Chazeu : Saône-et-Loire, arr. Autun, c. Mesvres, cne Laisy. Savoisy : Côte-d'Or, arr. Montbard, c. Laignes. Authumes : Saône-et-Loire, arr. Louhans, c. Pierre-de-Bresse. 25. Présilly (Jura, arr. Lons-le-Saulnier, c. Orgelet, cne Sarrogna) fouillé, restauré et étudié depuis 1996 par J.-J. Schwien. Perrigny (Côte-d'Or, arr. Dijon, c. Chenôve), fouillé de 1993 à 1996 par E. Laborier et H. Mouillebouche.

26. La Perrière : Saône-et-Loire, arr. Autun, c. Saint-Léger-sous-Beuvrey, cne Étang-surArroux. 
INDEX

Index géographique : France/Bourgogne

Mots-clés : château 